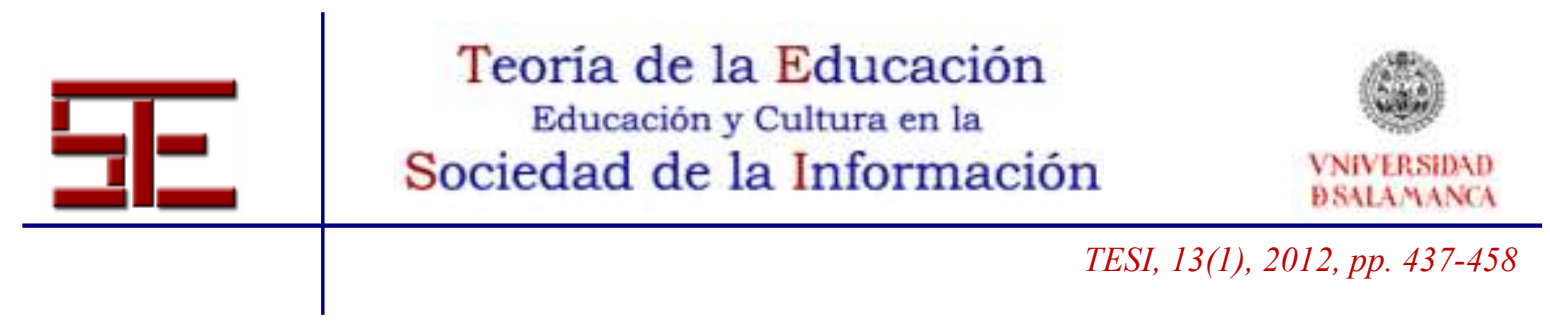

\title{
VALORACIÓN POR LOS ESTUDIANTES DE LA LICENCIATURA EN PSICOPEDAGOGÍA DE UNA EXPERIENCIA INNOVADORA BASADA EN ESTRATEGIAS DE APRENDIZAJE COLABORATIVO Y EL USO DEL COURSELAB
}

Resumen: Una sociedad en constante cambio tecnológico exige profesionales de la enseñanza en formación continua. Entre las principales competencias docentes que debe adquirir actualmente el profesorado, durante el proceso de formación inicial, podemos destacar el uso educativo de las Tecnologías de la Información y la Comunicación (TIC) y el trabajo en equipo. A través de esta experiencia de innovación centrada en el desarrollo de una dinámica de trabajo fundamentada en el aprendizaje cooperativo y la utilización del programa Courselab, se exigen al alumnado ciertas destrezas específicas en el dominio tanto del PC en su conjunto como de la navegación a través de Internet y de la capacidad de trabajo y de resolución en equipo. En definitiva, se pretende completar la formación de los futuros docentes en entornos TIC, para que puedan desempeñar procesos de enseñanza/aprendizaje que respondan, verdaderamente, a los intereses y motivaciones del alumnado y puedan contribuir a la mejora del éxito escolar. Los resultados de este estudio indican que estos profesores en formación, demandan el uso de herramientas similares que les motiven y se adecuen a los procesos de enseñanza aprendizaje, concluyendo con una valoración positiva tanto de la experiencia como del propio desarrollo de la asignatura.

Palabras clave: TIC; aprendizaje cooperativo; innovación; Courselab; estrategias de aprendizaje.

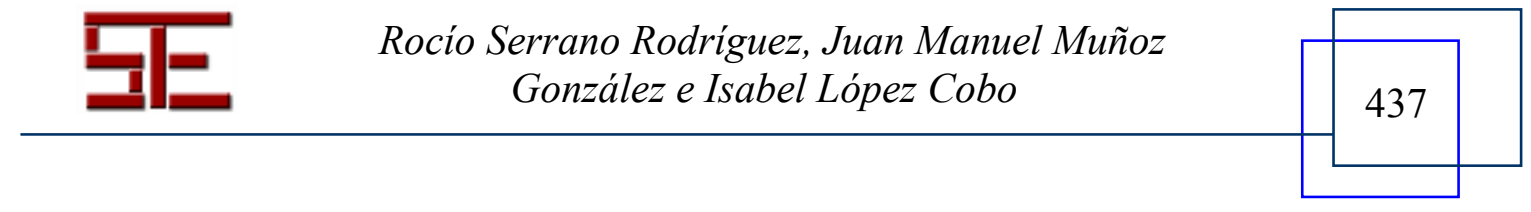




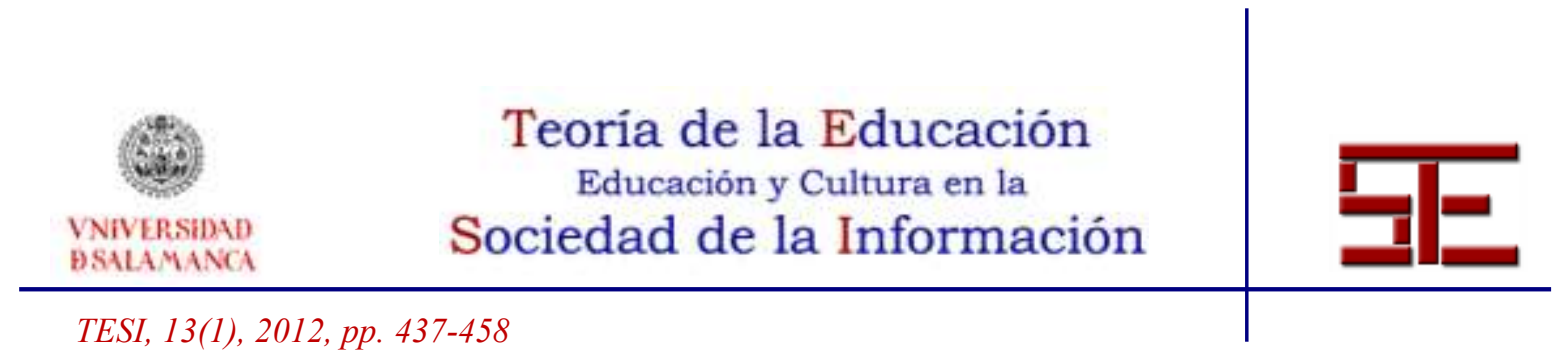

INNOVATIVE

EXPERIENCE EVALUATION

IN INITIAL HIGH $\begin{array}{lll}\text { SCHOOL TEACHER BASED IN COLLABORATIVE } & \text { LEARNING } \\ \text { STRATEGIES AND USE OF COURSELAB } & \end{array}$

Abstract: A technological society in permanent evolution requires teaching professionals with continuous training.

Among the main teaching skills that teachers must currently acquire during process of initial training, we emphasize the educational use of Information and Communication Technologies (in Spanish its acronym is TIC) and teamwork. Through this innovation experience focused on developing a workflow based on cooperative learning using CourseLab application, specific student skills are required in PC use, navigation through Internet and ability for teamwork and problem resolution. Definitely, we pretend to complete training of future teachers in ICT environments, so they could perform teaching / learning processes in order to match students interests and motivations, and may contribute to improvement of school success. Results of this study indicate that training teachers demand the use of similar tools to motivate themselves and adapt to teaching and learning processes. We conclude with a positive assessment of both experience and development of the subject.

Keywords: ICT; collaborative learning; innovation; Courselab; learning strategies.

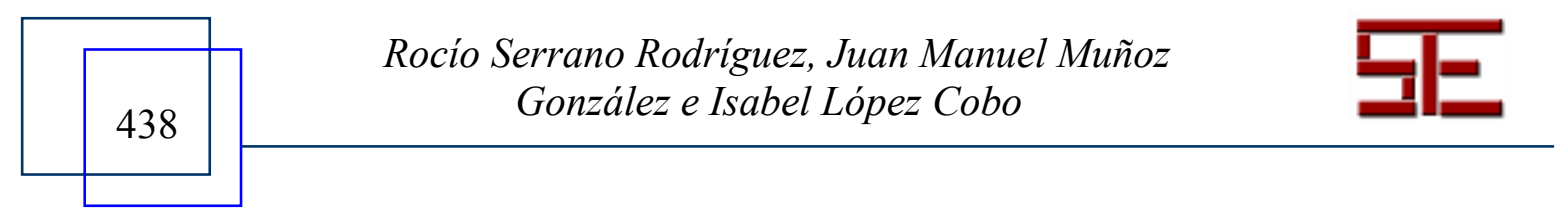




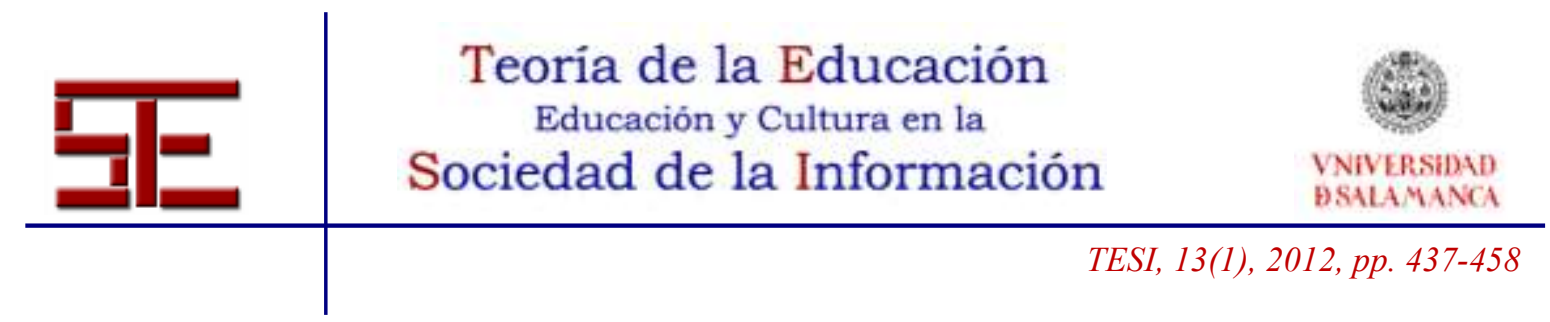

\section{VALORACIÓN POR LOS ESTUDIANTES DE LA LICENCIATURA EN PSICOPEDAGOGÍA DE UNA EXPERIENCIA INNOVADORA BASADA EN ESTRATEGIAS DE APRENDIZAJE COLABORATIVO Y EL USO DEL COURSELAB}

Fecha de recepción: 31/10/2011; fecha de aceptación: 27/02/2012; fecha de publicación: 00/03/2012

Rocío Serrano Rodríguez

rocio.serrano@uco.es

Universidad de Córdoba

Juan Manuel Muñoz González

juan.manuel@uco.es

Universidad de Córdoba

Isabel López Cobo

isabel.lopez@uco.es

Universidad de Córdoba

\section{1.- EL USO DE LAS TECNOLOGIAS DE INFORMACIÓN Y COMUNICACIÓN (TIC) EN LOS PROCESOS DE ENSEÑANZA- APRENDIZAJE}

Nos encontramos en una sociedad definida por muchos autores como la sociedad de la información y la comunicación. Esta sociedad se ha caracterizado en los últimos años por los grandes avances tecnológicos, avances que se producen cada vez de forma más vertiginosa y con mayor frecuencia.

Numerosos trabajos ponen de manifiesto la necesidad de incorporar el uso de las Tecnologías de Información y Comunicación (TIC) en los diversos niveles educativos y también en los procesos de formación inicial docente, desde el profesorado de educación infantil o primaria hasta la formación de profesores de secundaria $\mathrm{y}$ universidad (Cabero, Duarte y Barroso, 1997; Da Ponte, 2004; Peirano y Domínguez, 2008). En estos estudios, se demuestra el papel de las TIC como instrumentos que ayudan en la comunicación a través de diversos medios y que son necesarios para la

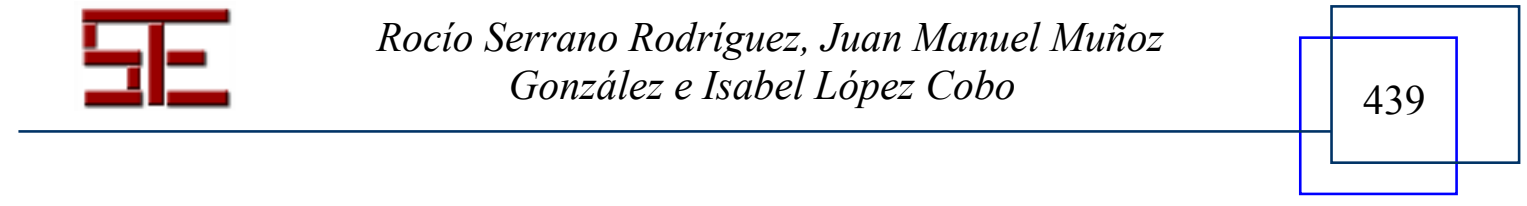




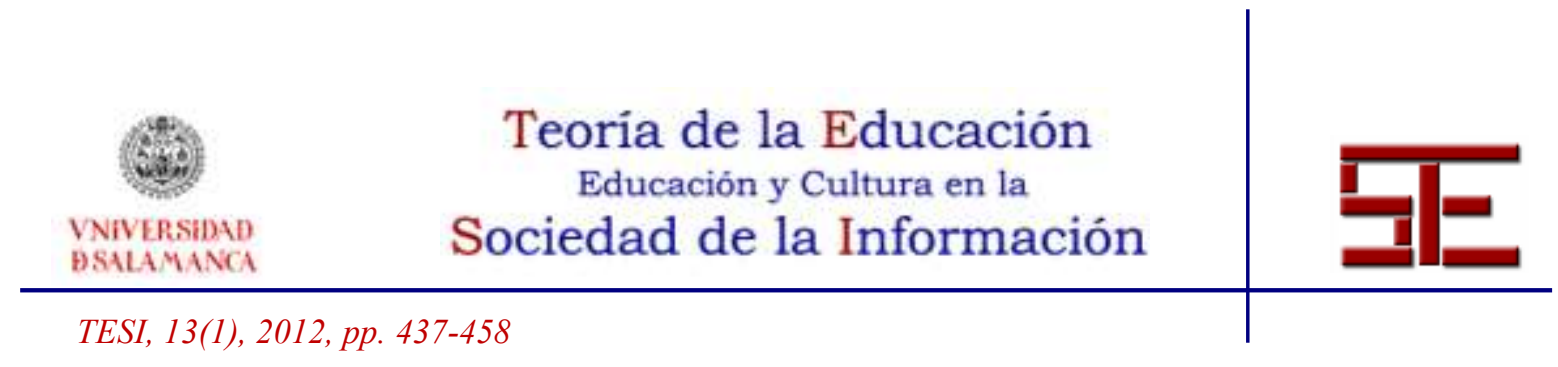

mejora de los procesos educativos en la sociedad actual (Sánchez, Lombardo, Riesco y Joyanes, 2004).

El uso de recursos educativos de este tipo facilita al profesorado en formación la consecución de una metodología activa e innovadora en sus clases, de tal manera que hacen operativos los nuevos planteamientos metodológicos del Espacio Europeo de Educación Superior (Cano, 2005). De ahí, surge el plantear nuevas estrategias de trabajo con nuestros discentes, en nuestro caso, a través del aprendizaje del programa Courselab y las dinámicas de aprendizaje colaborativo, como estrategias motivadoras para nuestro alumnado, tal y como puede observarse en los resultados obtenidos en esta investigación.

Así pues, las nuevas tecnologías se están convirtiendo en la base de nuevos escenarios de aprendizaje, dando lugar a la creación de nuevas situaciones y procesos de enseñanza (Cabero, Barroso y Llorente, 2008).

Estas herramientas educativas sirven también de base al fomento de la creatividad, al esfuerzo motivador, al ejercicio de la memoria y al desarrollo de numerosas aptitudes de tipo intelectual o práctico. En definitiva, las nuevas tecnologías proporcionan un recurso comunicativo fundamental para el acceso de todas las personas a la información y son sustento de habilidades diferentes, que pueden ser la base de aprendizajes diversificados, ligados a la creación de nuevas situaciones y procesos (Cabero, Barroso y Llorente, 2008).

En este sentido es hacia donde dirigimos nuestra investigación, pues a través del aprendizaje del programa Courselab, pretendemos crear una dinámica donde nuestro alumnado comprenda términos, funcionamiento y aplicación de herramientas novedosas en el ámbito educativo. Para ello, es necesario conocer el punto de partida o, lo que es lo mismo, conocer el programa Courselab y definir la dinámica de clase desarrollada.

\section{1.- El programa Courselab}

Comenzaremos este apartado explicando en qué consiste exactamente el programa Courselab, así como cuáles son sus principales funciones y aplicaciones en el ámbito educativo.

CourseLab es un software de creación de recursos multimedia que pueden ser empleados tanto con fines de e-learning, como en prácticas presenciales dentro del aula.

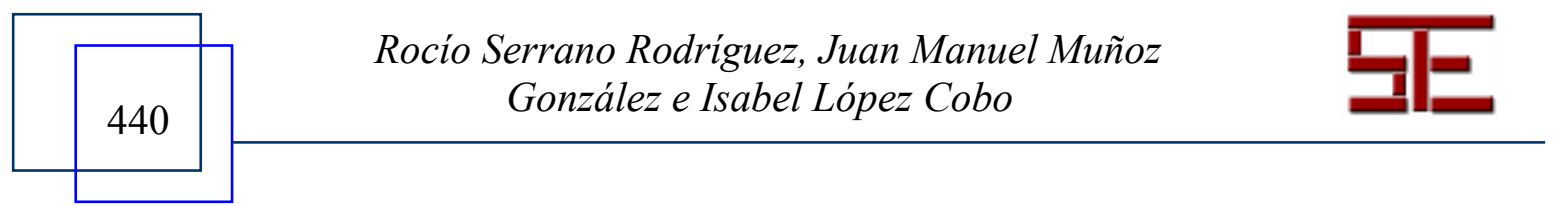




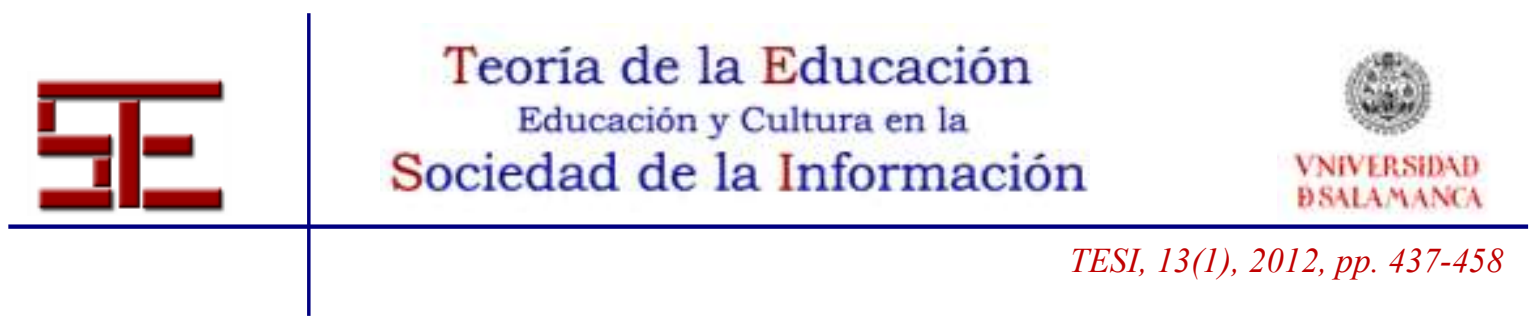

Constituye una herramienta muy potente y fácil de usar que ofrece la posibilidad de insertar contenidos de alta calidad (imágenes, vídeos, aplicaciones java, flash, etc.), así como la publicación de los resultados en diversos medios como Webs, Plataformas de Aprendizaje (Moodle, Dokeos, WebCT, etc.), CD-ROM, etc. Además de contenidos, nos proporciona la capacidad de embeber páginas web dentro de nuestros trabajos, desarrollar animaciones de forma intuitiva, así como crear distintos itinerarios de aprendizaje, de tal manera que nuestros materiales se adecuen al nivel de aprendizaje del alumnado. Por otra parte, encontramos, dentro de sus potencialidades, la integración de sistemas de evaluación automatizados, como test objetivos que pueden incorporar preguntas de distinto tipo; elección única, múltiple, respuesta numérica, emparejamiento, etc. Cuando hablamos de sistemas de evaluación automatizados, nos referimos a que estos test se corrigen de forma automática, dándonos el resultado en el mismo momento en el que se finalicen. Este tipo de función, combinada con las características propias de cualquier plataforma de aprendizaje que cumplan los estándares SCORM, nos aporta una evaluación detallada de cada uno de los alumnos/as que estén matriculados dentro de dicha plataforma y/o asignatura, pudiendo comprobar los resultados obtenidos en los distintos test que hayamos integrado en los posibles itinerarios de aprendizaje. Por último, conviene destacar que "No es cierto que colocar un contenido usando diapositivas, texto o vídeo conlleve a una verdadera instrucción. Tal contenido debe incluir recursos importantes para los estudiantes pero sólo podrán ser efectivos dentro de un contexto altamente motivante e interactivo" (Stahl, Koschmann y Suthers, 2006).

\section{2.- APRENDIZAJE COLABORATIVO}

Una vez definidas las características principales del programa Courselab, así como algunos de sus usos más habituales, nos adentramos en los principios que han sustentado el desarrollo de nuestra innovación a través del aprendizaje colaborativo como eje fundamental del proceso de enseñanza-aprendizaje. Por ello, debemos hacer mención a las características que el Espacio Europeo de Educación Superior (EEES) considera en los títulos universitarios en relación a los resultados del aprendizaje y, esencialmente, al desarrollo de competencias, quedando reflejado de manera implícita en algunas de ellas la competencia de «aprender a aprender» en un entorno participativo, caracterizado por el trabajo en equipo y la implicación responsable del alumnado en su propio proceso de aprendizaje.

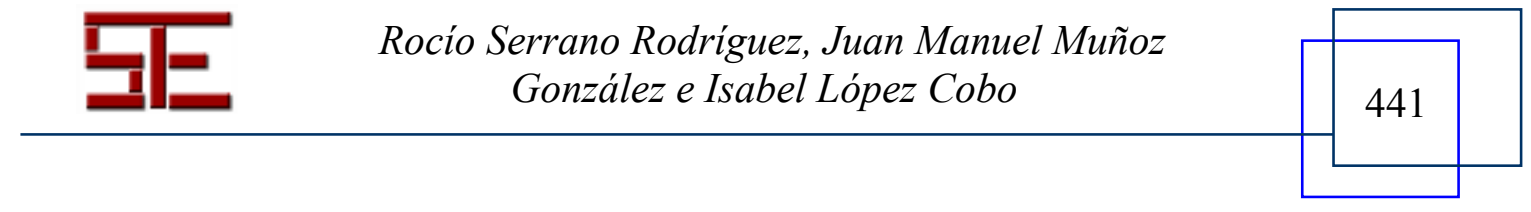




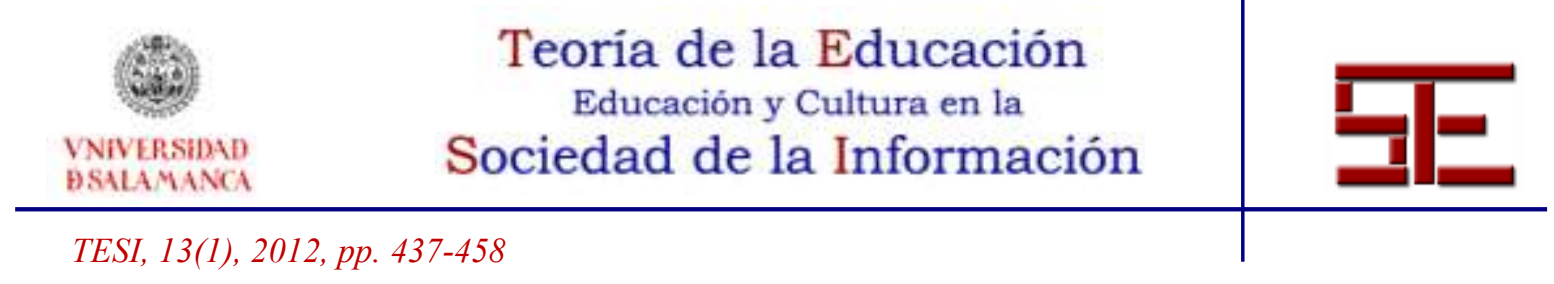

Tal y como afirman Gisbert, Cela-Ranilla e Isus (2010) es posible enseñar estas competencias transversales en una acción conjunta que implique:

- La incorporación de metodologías docentes que permitan desarrollar las competencias desde la programación de cada asignatura o módulo.

- La implicación práctica en empresas y entornos laborales.

- Y las situaciones simuladas de entornos profesionales, que faciliten la transferencia de conocimientos y actitudes en la práctica profesional.

El desarrollo de proyectos colaborativos es una de las posibles estrategias metodológicas válidas para poner en práctica este particular enfoque de la educación, puesto que permite al alumnado aprender conjuntamente y compartir tanto responsabilidades como logros. En este sentido, y como afirma John (1998), el aprendizaje colaborativo es, ante todo, un sistema de interacciones cuidadosamente diseñado, que organiza e induce la influencia recíproca entre los integrantes de un equipo. Es también un proceso en el que se va desarrollando gradualmente, entre los integrantes de dicho equipo, el concepto de ser "mutuamente responsables del aprendizaje de cada uno de los demás". Ovejero (1990), por su parte, añade a esto: "en el aprendizaje colaborativo debe tenerse en cuenta el principio general de intervención, que consiste en que un individuo solamente adquiere sus objetivos si el resto de los participantes adquieren el suyo; no se refiere, por tanto, al simple sumatorio de intervenciones, sino a la interacción conjunta para alcanzar objetivos previamente determinados".

\section{1.- Características del aprendizaje colaborativo}

Carrió (2007), Rosario (2008) y Echazarreta (2009) exponen algunas de las numerosas características que identifican y diferencian un aprendizaje colaborativo de otros tipos de aprendizaje:

- Orgullo de pertenecer al grupo: El grupo ha de reconocerse como tal y estar convencido de que el reparto de tareas es equitativo para ello la comunicación entre los miembros ha de ser fluida y las bases del liderazgo compartido han de ser conocidas y aceptadas por todos.

- Heterogeneidad de los componentes del grupo: La formación de los grupos en el trabajo colaborativo es diversa en habilidades y características de los miembros, lo cual permite que los distintos puntos de vista de los componentes del grupo generen unas conclusiones más complejas y ricas. Así, encontramos que el

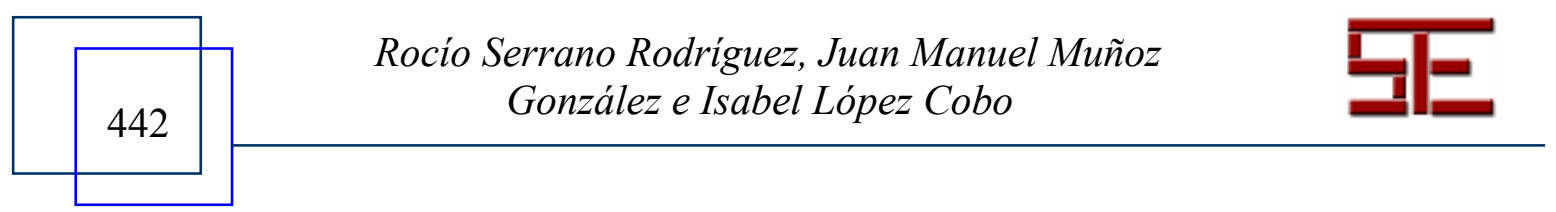




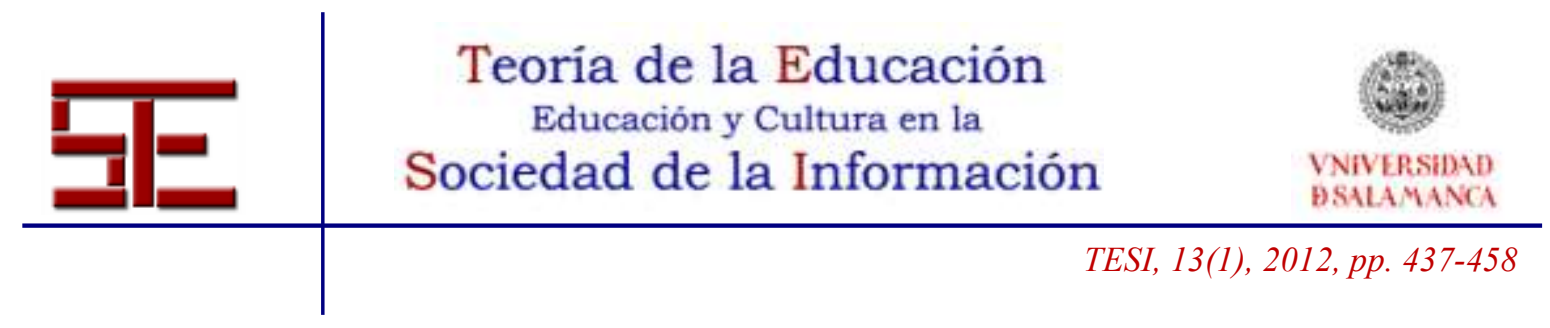

consenso de las conclusiones a las que llega el grupo parte de puntos de vista distintos y con ello las aportaciones finales han sido discutidas, por lo que el aprendizaje es activo desde un punto de vista sociocognitivo permitiendo esbozar opciones que el aprendizaje individual o liderado por un solo individuo no hubiera producido.

- Interdependencia de los miembros que lo conforman: Esta característica está relacionada con la anterior en el sentido de que hay una clara responsabilidad individual de cada miembro del grupo para el alcance de la meta final.

- Intersubjetividad de los conocimientos: Se comprende e interpreta un tema de forma compartida, por lo que los puntos de vista subjetivos se intercambian y discuten hasta obtener uno solo.

- Liderazgo individual: Cada miembro del grupo es su propio líder y ha de responder por las cuestiones que le son encomendadas por el grupo, lo que favorece el refuerzo individual de los propios conocimientos y argumentaciones.

- Aparición y soluciones de los conflictos grupales: Es necesario contemplar la aparición de conflictos grupales que surgen, en parte, por el intercambio de formas de pensar diferentes y por la propia heterogeneidad del grupo. Es por ello que hemos de tener presente esta posibilidad para poder llegar a soluciones adecuadas y que no lleven al grupo a crear subgrupos o líderes enfrentados, ya que de ser así la dinámica del grupo se convertiría en un aprendizaje grupal o individualizado. En consecuencia, el trabajo colaborativo exige a los participantes: habilidades comunicativas, relaciones simétricas y recíprocas y deseo de compartir la resolución de tareas.

\section{2.- El uso de las TIC en el desarrollo del aprendizaje colaborativo}

La innovación que presentamos en este artículo ha mostrado tener gran eficacia para aumentar la motivación, mejorar el rendimiento, potenciar las habilidades sociales y desarrollar las capacidades de aprendizaje haciendo uso del aprendizaje colaborativo.

Indudablemente, el desarrollo de proyectos colaborativos puede experimentar un notable impulso con la utilización de las modernas tecnologías de la información y de la comunicación. El uso de las TIC facilita tanto la búsqueda y el procesamiento de información como el desarrollo original de contenidos por parte del alumnado, siendo a la vez una excelente herramienta para atender la diversidad y promover la comunicación y la expresión creativa.

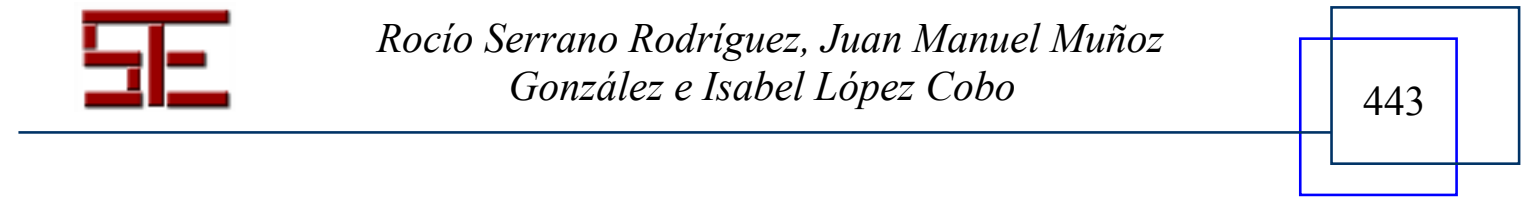




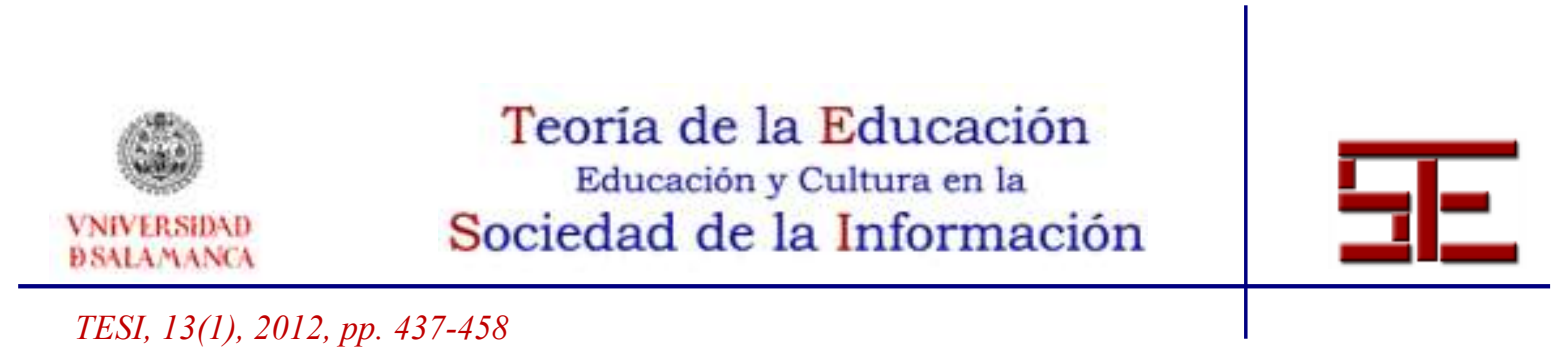

Según Echazarreta, Prados, Poch y Soler (2009) el éxito de un proyecto colaborativo depende en buena medida de una adecuada planificación y estructuración previa, que articule tanto los objetivos que se pretenden alcanzar como los contenidos del proyecto y la metodología que se va a aplicar en su desarrollo.

En este sentido, conviene prestar especial atención a algunos aspectos como son la disponibilidad de los recursos, la organización coherente de los agrupamientos, la distribución temporal de las actividades o los mecanismos de seguimiento y evaluación que nos permitirán reflexionar sobre el desarrollo del proyecto y reorientarlo según las necesidades que vayamos detectando.

Otro aspecto de gran interés a la hora de alcanzar el objetivo marcado por medio del aprendizaje colaborativo es el hecho de que el profesorado deberá estar preparado para preservar al máximo los beneficios que le posibilitan las tecnologías en cuanto que: favorecen escenarios de aprendizaje distintos centrados en el alumnado, les brindan diversas modalidades de interacción, les aportan contextos y modos de seguimiento de su propio proceso de aprendizaje, parten de sus intereses personales y desarrollan una mayor autonomía de trabajo y aprendizaje autorregulado (Area, 2005). En este sentido, y tal y como afirman Morales, Díaz y García (2011) "el diseño de materiales educativos orientados al desarrollo de competencias cobra hoy en día una especial importancia".

Debido a lo anteriormente expuesto, consideramos que la aplicación que presentamos en este artículo, favorece por un lado, la nueva labor del docente del siglo XXI y por otro, potencia el desarrollo y adquisición de algunas de las competencias reflejadas en las nuevas titulaciones universitarias. En este sentido, nos estamos refiriendo a la aplicación por parte de los futuros profesores de herramientas informáticas que contribuyen notablemente a mejorar el aprendizaje significativo de los contenidos educativos del proceso de formación y a desarrollar algunas de las competencias, tales como: familiarización con las TIC, trabajo en equipo y metacognición o reflexión sobre los propios conocimientos del profesorado en formación (González, 2008).

No cabe duda, de que aplicar una metodología que apuesta por la realización de proyectos colaborativos favorece el desarrollo cognitivo de toda la comunidad de aprendizaje (Villalustre y Del Moral, 2011), pero si a esto le unimos el uso de una herramienta que mejora la representación del conocimiento, la organización, la

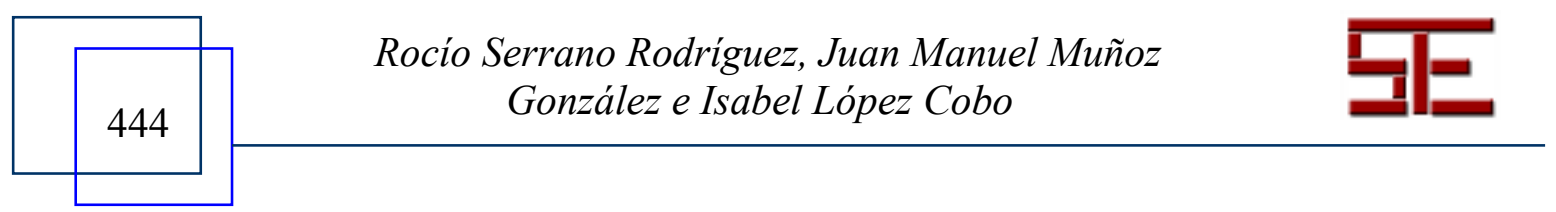




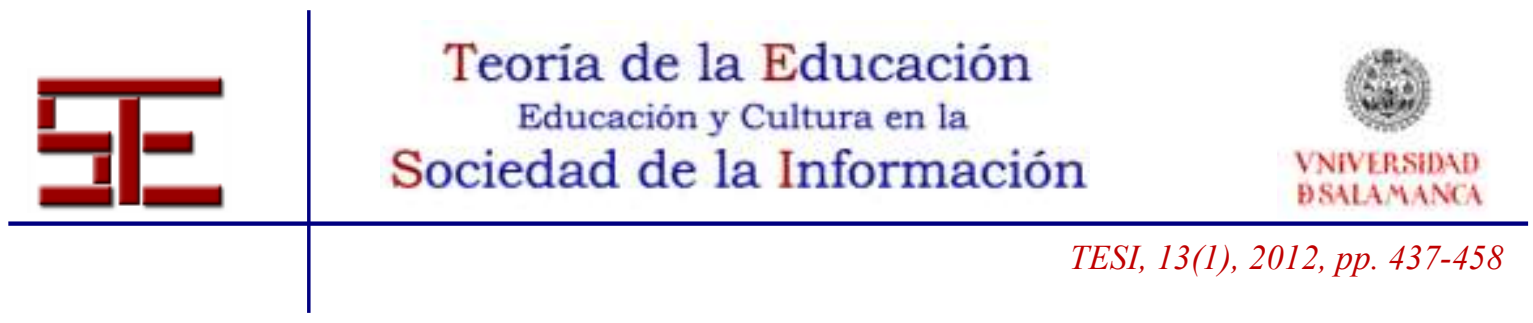

adquisición de contenidos y el desarrollo personal y académico, nos encontramos frente a un uso de las TIC que posibilita una enseñanza más flexible y abierta.

\section{3.- DESARROLLO DE LA DINÁMICA DE APRENDIZAJE: UNA EXPERIENCIA INNOVADORA}

A continuación, describiremos el contexto de la dinámica de aprendizaje así como todas y cada una de las fases de la misma desarrolladas en el aula y que permitirá clarificar todo el proceso de enseñanza/aprendizaje, así como su conexión con los objetivos propuestos en la investigación.

\section{1.- Contexto}

La experiencia de innovación docente se ha desarrollado en la asignatura de Diseño, desarrollo e innovación del currículum, asignatura troncal del plan de estudios de la Licenciatura de Psicopedagogía de la Facultad de Ciencias de la Educación de la Universidad de Córdoba (España).

El contexto en el que se ha desarrollado ha sido el aula TIC de la Facultad de Ciencias de la Educación de la Universidad de Córdoba. Pese a que el aula estaba equipada de equipos suficientes para que cada alumno/a pudiera llevar a cabo el aprendizaje de manera individual, se permitió la utilización de ordenadores portátiles propios, facilitando su conexión a la red tanto eléctrica como wifi o inalámbrica a través de la propia infraestructura del aula.

\section{2.- Equipamiento}

Los equipos instalados en dicha aula poseen los Sistemas Operativos más usados hoy día, como son Linux y Microsoft Windows, siendo este último indispensable para la ejecución de Courselab. Estos, a su vez, actúan como equipos remotos dependientes de un servidor donde se encuentran instaladas todas las aplicaciones que utilizan los usuarios del centro, por lo que el alumnado dispone de un espacio limitado asociado a su sesión de usuario, aspecto que limita, en cierta medida, el desarrollo de la dinámica, como veremos a continuación.

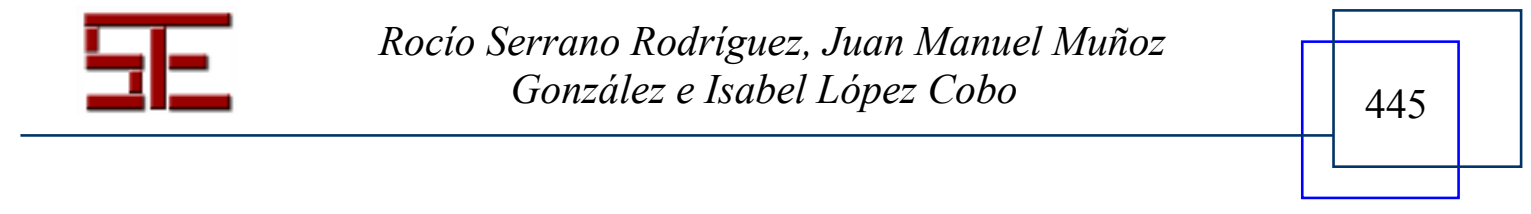




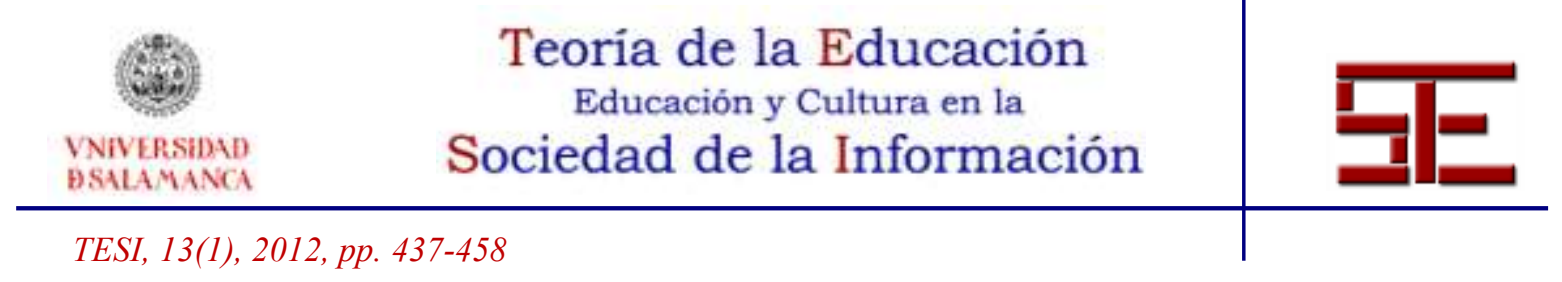

\section{3.- Plataforma de aprendizaje y estándar SCORM}

La plataforma de aprendizaje de la Universidad de Córdoba es Moodle, una aplicación de Código Abierto denominada también Sistema de Gestión del Aprendizaje (Learning Management System o LMS). Una de las grandes ventajas de esta plataforma es que es compatible con los estándares SCORM. El módulo SCORM de la plataforma nos permite cargar fácilmente cualquier paquete SCORM (Sharable Content Object Reference Model) y convertirlo en parte de un curso. Es aquí donde se dirige nuestra dinámica de aprendizaje, pues el resultado de la misma será el desarrollo de recursos didácticos multimedia que sean compatibles con dichos estándares.

\section{4.- Dinámica de aprendizaje}

El proceso de enseñanza y aprendizaje llevado a cabo comprende varias fases:

$1^{a}$. Introducción. Objetivos, guía orientativa y ejemplificación de la práctica: En primer lugar, se ha considerado indispensable clarificar cuáles son los objetivos que se pretende conseguir con la práctica que el alumnado tiene que llevar a cabo dentro de la asignatura. Para ello, se ha entregado un documento explicativo en el que aparece el proceso que vamos a seguir tanto para el aprendizaje del programa como para la realización concreta de la práctica. Por último, el profesor muestra y expone un ejemplo de una práctica ya realizada en el que el alumno observa cuál será el resultado del trabajo que deberá realizar con el resto de compañeros/as.

$2^{a}$. Fase previa. Requisitos, descarga e instalación del programa: Conviene que el alumnado conozca cuáles son los requisitos para la ejecución del programa, aspecto que se puede observar en la web del mismo. Aunque, anteriormente, hemos comentado que los equipos contienen el programa, es necesario que el alumnado conozca la WEB donde se aloja, así como el procedimiento para la descarga e instalación del mismo. Pese a que las características, en cuanto a hardware, son muy básicas y permiten su ejecución en, prácticamente, cualquier ordenador del mercado (PC y no MAC), es necesario que el alumnado conozca aquellos componentes que se consideran indispensables para un buen funcionamiento del mismo, como son Adobe Flash Player y Javascript. Estos complementos son totalmente gratuitos, por lo que, dentro de esta dinámica, también procedemos a la localización de las webs de los mismos, descarga e instalación.

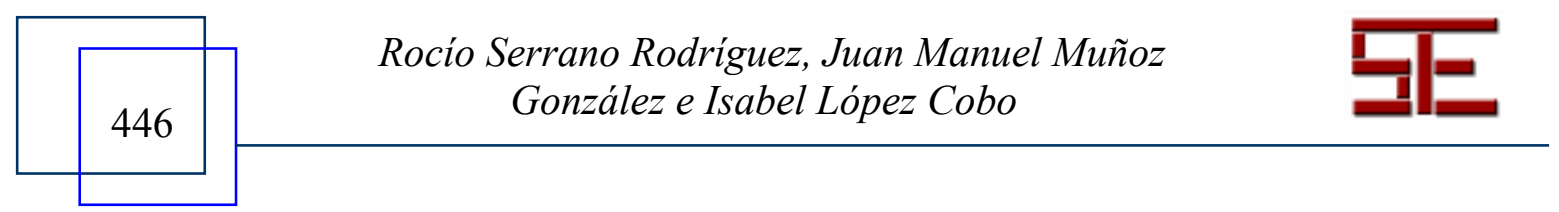




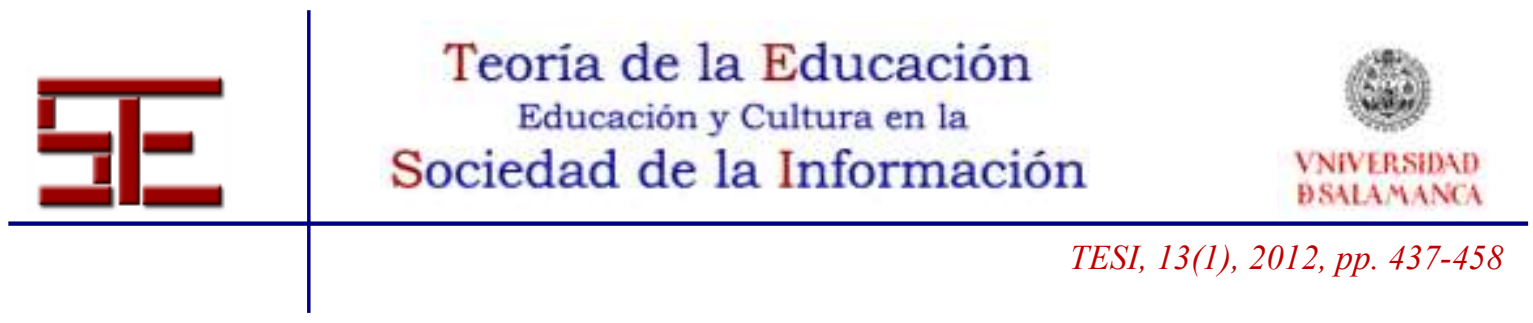

$3^{a}$. Fase inicial. Familiarización con el entorno de la aplicación: Una vez que el alumnado ya ha descargado e instalado el programa y los componentes mencionados (sólo aquel que ha usado su propio portátil), continuamos con la ejecución de la misma y la familiarización con su entorno o "interface". Este es muy parecido a los usados por las aplicaciones de Microsoft pertenecientes al paquete Office, por lo que, de manera prácticamente intuitiva, el alumnado conoce la situación y funcionalidad de las distintas barras de tareas e iconos de acceso rápido, haciendo especial hincapié en la funcionalidades de las distintas áreas de trabajo del programa.

$4^{a}$. Fase principal. Aprendizaje colaborativo: Concluida la fase de familiarización, se ha continuado con el aprendizaje de las distintas funciones que ofrece el programa y su conexión con los objetivos y la guía de la práctica. En esta fase resulta de especial relevancia la división del tiempo en tres partes: la primera parte, se destina a la explicación y demostración de las distintas funciones por parte del profesor. Seguidamente, el alumnado, una vez que se ha explicado una función, realiza el proceso de manera individual y se procede a la resolución de dudas o dificultades. El último espacio de tiempo de la clase es el destinado a que cada grupo, tomando como referencia la guía orientativa de la práctica, ponga en funcionamiento los conocimientos adquiridos y resuelva las posibles dificultades de manera consensuada y en equipo.

$5^{a}$. Fase Final: En esta fase, ya se ha concluido la explicación del programa y su principal objetivo es el desarrollo, resolución de dificultades, conclusión y exposición de la práctica de cada uno de los grupos. Esta requiere que cada grupo haya publicado la práctica en formato SCORM y la haya subido a la plataforma de aprendizaje. Esta fase concluye con una valoración grupal de los trabajos presentados por el resto de grupos de trabajo.

\section{4.- PROCESO DE INVESTIGACIÓN}

\section{1.- Objetivos}

La hipótesis principal de este estudio, sustentada en la revisión de la literatura que se ha expuesto anteriormente, es la siguiente: "Los futuros profesores que utilizan la aplicación de programas como el Courselab dentro de una dinámica de trabajo fundamentada en el aprendizaje colaborativo para la adquisición de contenidos durante el proceso de formación inicial, adquieren destrezas útiles para aplicar estas

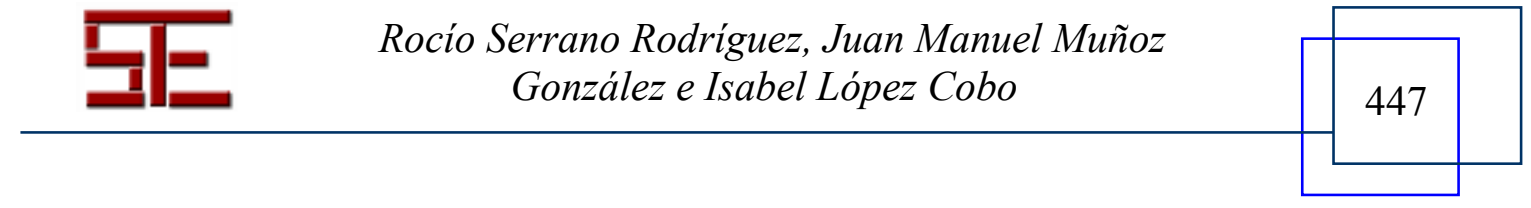




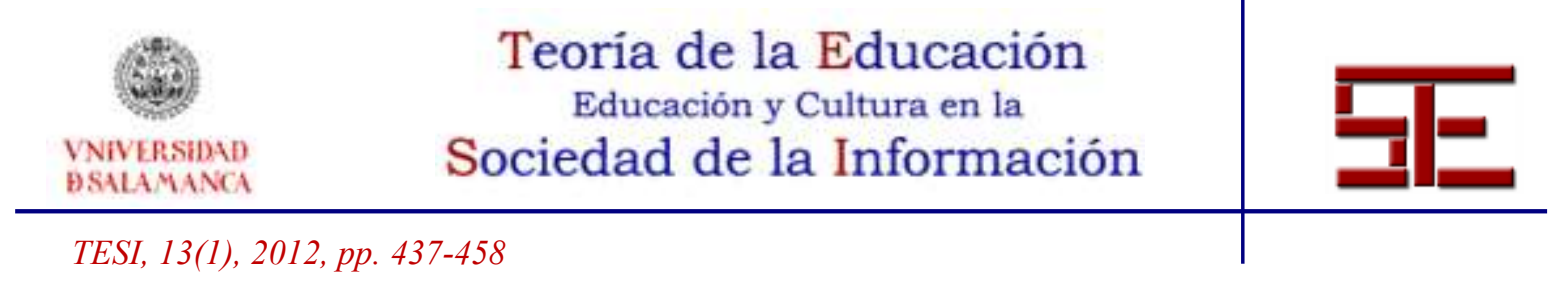

herramientas en su futuro contexto como docentes valorando favorablemente la utilización de tales recursos como instrumentos de aprendizaje colaborativo".

Indicar que la finalidad de nuestra dinámica radica en que nuestro alumnado, concebido como futuros profesionales en el ámbito de la enseñanza, necesita dominar herramientas que faciliten los procesos de enseñanza-aprendizaje, a través de materiales que respondan a los intereses y motivaciones de su alumnado, tratando de mejorar la calidad de los procesos de enseñanza-aprendizaje de los contenidos específicos de la asignatura de Diseño, desarrollo e innovación del currículum, planteando la consecución de los siguientes objetivos en el desarrollo de la innovación:

- Aprender el uso del programa Courselab, como una estrategia que puede ser muy útil a los futuros profesores, tanto en su actividad docente y comunicativa, como en el diseño de actividades a realizar por sus alumnos en el aula.

- Fomentar la actitud reflexiva, desarrollando la metacognición y el pensamiento crítico acerca del conocimiento que se va adquiriendo durante el proceso de formación inicial docente, incluyendo la reflexión sobre las estrategias y los recursos utilizados en la construcción de dicho conocimiento.

- Adquirir destrezas en el uso educativo de las TIC, aprendiendo a manejar el software.

- Favorecer la interacción social en el aula, fomentando la capacidad de trabajo en equipo y el aprendizaje colaborativo.

Esta experiencia forma parte de un proyecto de trabajo más amplio, cuyo eje común es el desarrollo de competencias docentes en los procesos de formación inicial del profesorado, utilizando recursos TIC considerados como estrategias de aprendizaje reflexivo y colaborativo. Tales recursos se están utilizando actualmente en diversas materias de los planes de estudio de magisterio y del máster de profesorado de enseñanza secundaria de nuestra universidad. Aunque los contenidos de tales materias son diferentes y se trata de colectivos de alumnos distintos, existe una metodología de trabajo docente común y unas metas.

Por ello, en este artículo nos centraremos únicamente en dar respuestas a estos tres objetivos:

1. Conocer la valoración que nuestro alumnado realiza de la experiencia y del desarrollo de la asignatura.

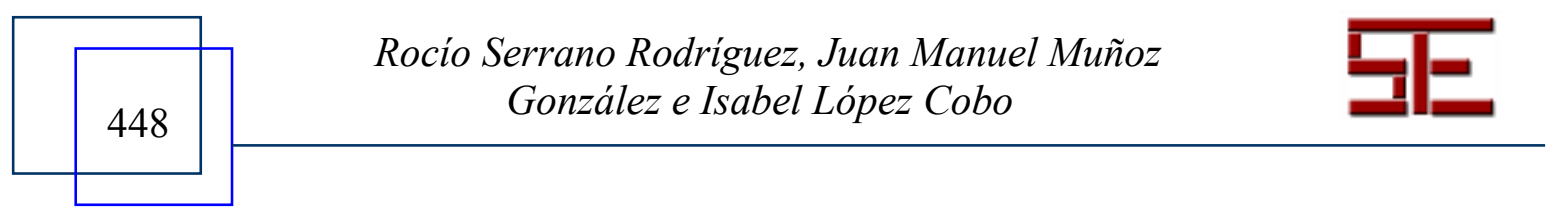




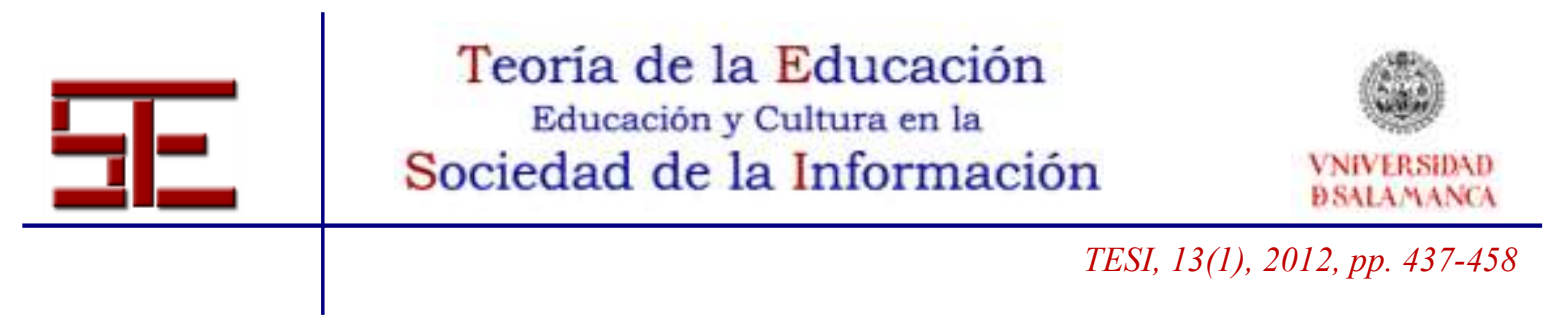

2. Indagar sobre las futuras utilidades de la aplicación de Courselab como futuros docentes.

3. Analizar las conclusiones de la puesta en marcha de una dinámica de aprendizaje colaborativo.

\section{2.- Descripción de la muestra}

La muestra comprende 44 alumnos/as universitarios pertenecientes al primer curso de la titulación de Licenciatura en Psicopedagogía, concretamente al grupo de tarde. El $63,3 \%$ de la muestra eran mujeres y el 36,4\% restante eran hombres. Destacando por su situación laboral activa un 59,1\% (26) de la muestra total encuestada.

La experiencia se ha llevado a cabo durante el curso académico 2010/2011.

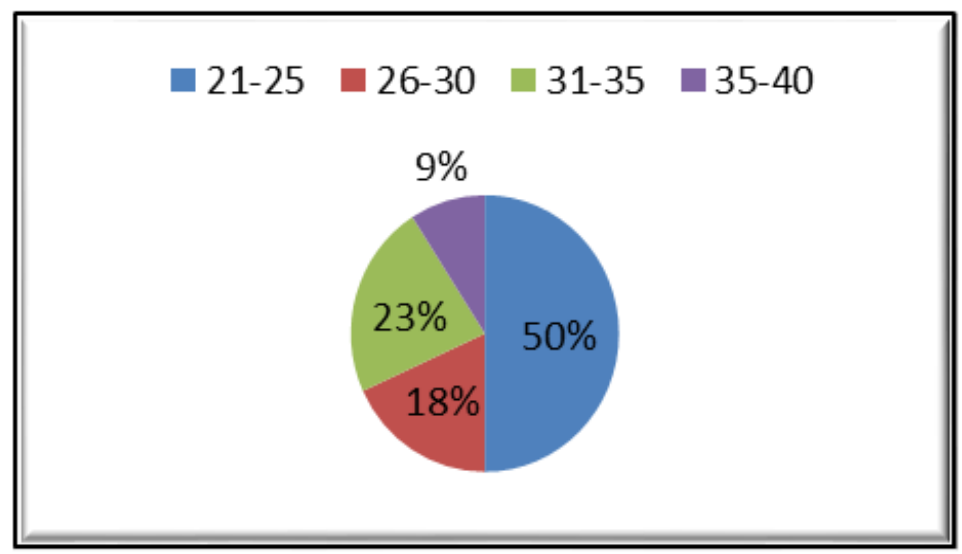

Gráfico 1. Distribución de la población encuestada por edades.

\section{3.- Método}

El trabajo se enmarca dentro de la metodología de la investigación cuantitativa (León \& Montero, 1997).

Como hemos indicado anteriormente, en este estudio sólo se analizan los datos correspondientes a los objetivos planteados y que se corresponden con el último bloque del cuestionario completo denominado "Cuestionario sobre competencias TIC para el alumnado universitario" (Muñoz y Serrano, 2011), compuesto por un total de 34 ítems.

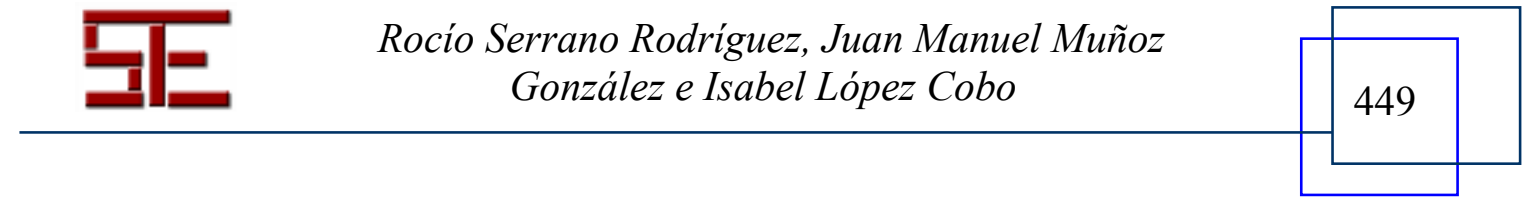




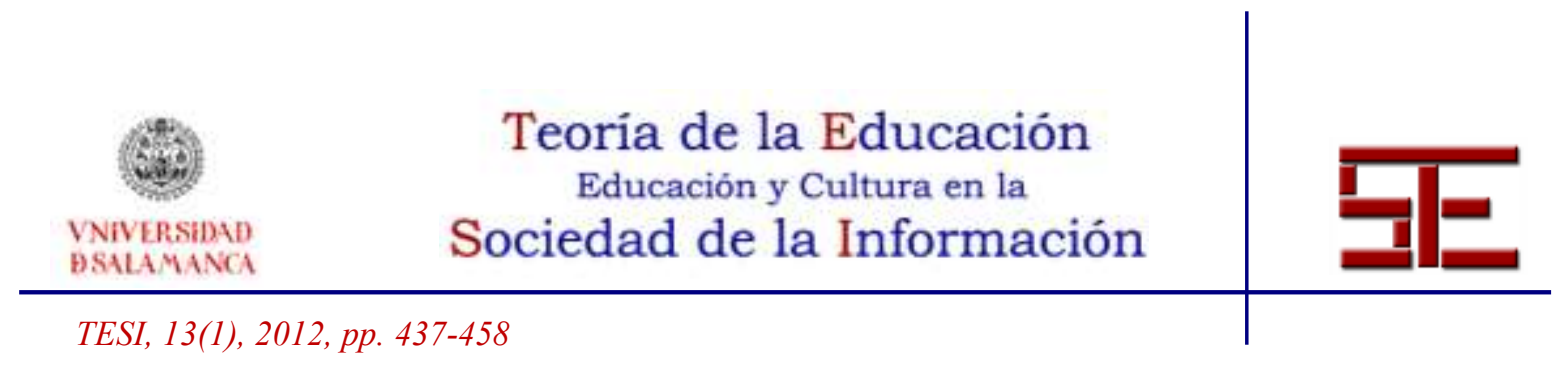

Para analizar las opiniones de los alumnos, acerca del uso del Courselab y el aprendizaje colaborativo, se han seleccionado las proposiciones correspondientes a: la utilidad de la aplicación práctica del Courselab, intereses y motivaciones y opiniones sobre la dinámica de aprendizaje colaborativo.

Los alumnos y alumnas debían valorar el grado de acuerdo o desacuerdo con cada una de las proposiciones con respuestas dicotómicas bajo las consignas "sí o no", o varias opciones de respuesta concretas.

Los resultados de las primeras secciones del test se expondrán en otros trabajos posteriores, pero por limitaciones de espacio en este estudio sólo se analizan los datos correspondientes a la última sección del cuestionario, integrada por 24 ítems.

Con ello se pretende recoger datos destinados a valorar si, a través del método docente aplicado, del recurso TIC utilizado y de la dinámica de aprendizaje colaborativo, el alumnado valora positivamente la innovación metodológica como favorecedora del proceso de enseñanza-aprendizaje.

\section{5.- RESULTADOS}

El cuestionario se implementó al finalizar el curso, de forma individual, por parte de todos los alumnos y alumnas de cada grupo que asisten habitualmente a clase presencial. Tras la recogida y codificación de datos, se procedió al análisis de los mismos usando el paquete estadístico SPSS (Versión 18.0).

Por otra parte, se ha realizado un estudio de fiabilidad del instrumento con los datos de toda la muestra, obteniendo en la primera de sus dimensiones un coeficiente de alpha de Crombach de 0,84. En la segunda dimensión del estudio, referida a los datos que se presentan en este artículo, hemos obtenido un coeficiente de alpha de Crombach de 0,77 , considerándolo suficiente en este estudio preliminar, teniendo en cuenta que el cuestionario original constaba de 34 ítems y, tras su depuración, se han eliminado 10 variables mediante el método de extracción: Análisis de componentes principales.

Por otra parte, la validación del cuestionario se ha realizado mediante expertos, lo que conllevó la reelaboración de algunos de sus ítems, así como una reestructuración del mismo.

A continuación presentamos los resultados en función a dos criterios. Por una parte, los aspectos vinculados a la valoración de la metodología y el desarrollo de la asignatura y,

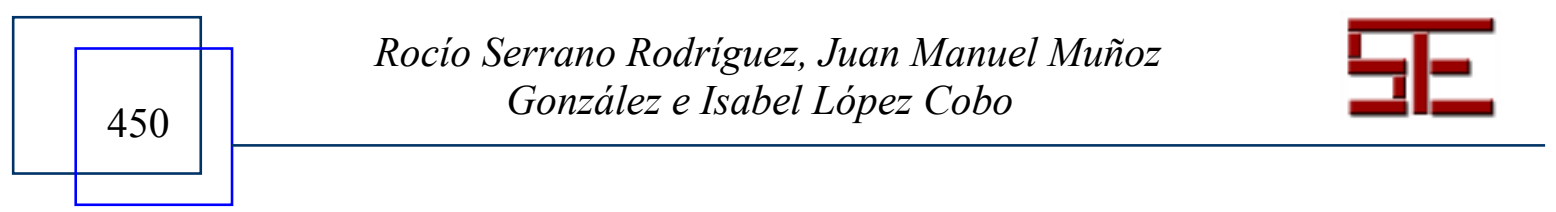




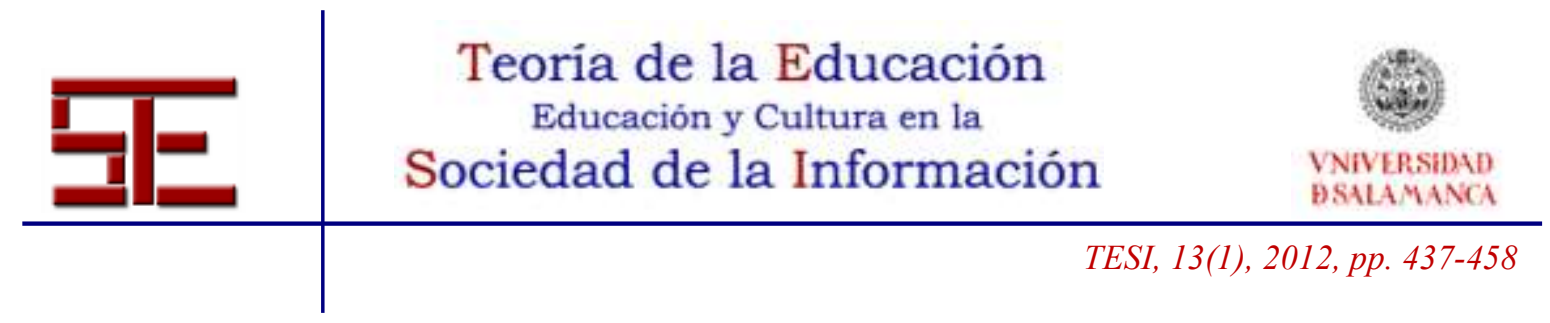

por otro lado, los resultados vinculados al desarrollo del aprendizaje colaborativo, aunque finalmente se realizará una discusión conjunta de tales resultados.

\section{1.- Valoración de la metodología y el desarrollo de la asignatura}

En primer lugar, se ha realizado un análisis descriptivo de frecuencias y porcentajes para los valores de los diferentes ítems del cuestionario en el conjunto de la muestra, cuyos resultados aparecen en la Tabla 1.

\begin{tabular}{|l|c|c|c|c|}
\hline \multicolumn{1}{|c|}{ Ítems } & \multicolumn{2}{|c|}{ Frecuencia } & \multicolumn{2}{c|}{$\begin{array}{c}\text { Porcentaje } \\
(100 \%)\end{array}$} \\
\cline { 2 - 5 } & Sí & No & Sí & No \\
\hline $\begin{array}{l}\text { ¿El uso de las TIC en el desarrollo de la asignatura ha } \\
\text { favorecido tu proceso de enseñanza? }\end{array}$ & 44 & 0 & 100 & 0 \\
\hline ¿El uso del Courselab ha mejorado tú formación? & 36 & 8 & 81,8 & 18,2 \\
\hline ¿Te ha resultado motivadora la asignatura? fel uso del & 26 & 18 & 59,1 & 40,9 \\
\hline $\begin{array}{l}\text { ¿Te ha resultado fácil de aprender el uso } \\
\text { Courselab? }\end{array}$ & 22 & 50 & 50 \\
\hline $\begin{array}{l}\text { ¿Crees que el uso de las TIC puede perjudicar tu } \\
\text { distracción en la adquisición de contenidos teórico? }\end{array}$ & 24 & 20 & 54,4 & 45,5 \\
\hline $\begin{array}{l}\text { ¿Consideras el número de horas dedicado a la } \\
\text { asignatura adecuado? }\end{array}$ & 10 & 34 & 22,7 & 77,3 \\
\hline $\begin{array}{l}\text { ¿Te gustaría haber trabajado contenidos relacionados } \\
\text { con Windows 7? }\end{array}$ & 32 & 12 & 72,7 & 27,3 \\
\hline ¿Te gustaría haber abordado contenidos de ofimática? & 4 & 40 & 9,1 & 90,9 \\
\hline $\begin{array}{l}\text { ¿Te gustaría haber conocido otras aplicaciones } \\
\text { educativas? }\end{array}$ & 36 & 8 & 81,8 & 18,2 \\
\hline $\begin{array}{l}\text { ¿Consideras necesaria la formación en otras } \\
\text { asignaturas relacionada con las TIC? }\end{array}$ & 44 & 0 & 100 & 0 \\
\hline ¿Crees que has consolidado bien toda la información? & 14 & 30 & 31,8 & 68,2 \\
\hline $\begin{array}{l}\text { ¿El desarrollo de esta metodología ha aumentado mi } \\
\text { interés personal por la temática? }\end{array}$ & 26 & 18 & 59,1 & 40,9 \\
\hline $\begin{array}{l}\text { ¿Crees que metodologías como la seguida en la } \\
\text { asignatura mejoran tu calidad como futuro profesional } \\
\text { docente? }\end{array}$ & 36 & 8 & 81,8 & 18,2 \\
\hline ¿Utilizarías el Courselab para actividades de refuerzo & 28 & 16 & 63,6 & 36,4 \\
\hline
\end{tabular}

Docio Serrano Rodríguez, Juan Manuel Muñoz González e Isabel López Cobo 


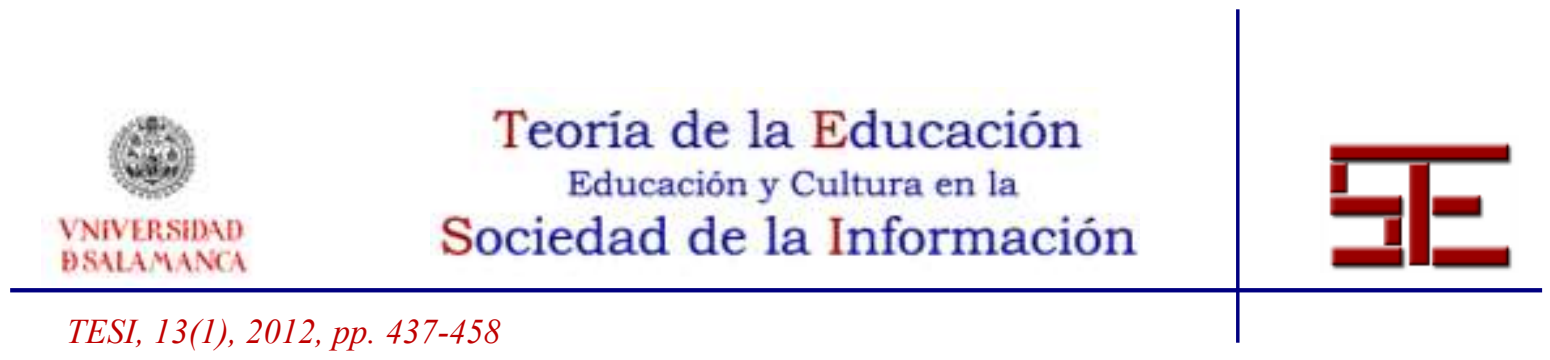

\begin{tabular}{|l|c|c|c|c|}
\hline y ampliación? & & & & \\
\hline $\begin{array}{l}\text { En general, ¿crees que la metodología seguida ha sido } \\
\text { adecuada? }\end{array}$ & 42 & 2 & 95,5 & 4,5 \\
\hline $\begin{array}{l}\text { ¿La organización del espacio ha favorecido el } \\
\text { desarrollo adecuado de la dinámica? }\end{array}$ & 14 & 30 & 31,8 & 68,2 \\
\hline
\end{tabular}

Tabla 1: Frecuencias y porcentajes sobre la metodología y el desarrollo de la asignatura

Se observa en la mayoría de las preguntas una valoración positiva en la metodología y el desarrollo de la asignatura, excepto en consideran que las TIC pueden ser un elemento distractor en la adquisición de contenidos $(54,4 \%)$, apuntan que determinados conceptos no se han consolidado $(68,2 \%)$ y el bajo interés por abordar contenidos de ofimática $(90,9 \%)$.

Los mayores porcentajes inciden en el uso de las TIC como favorecedoras de los procesos de enseñanza-aprendizaje (100\%) y la necesidad de nuevas asignaturas relacionadas con las TIC (100\%). Es destacable que el 95,5\% del alumnado valore como adecuada la metodología seguida en la asignatura y el uso de Courselab como una herramienta que ha mejorado su formación docente $(81,8 \%)$, aunque el $50 \%$ del alumnado considera que ha tenido dificultades en el dominio de este software. Debe matizarse también que, un elevado porcentaje $(77,7 \%)$ resalta la necesidad de un mayor número de horas para el desarrollo de esta asignatura.

Finalmente indicar que un $68,2 \%$ del alumnado encuestado opina que la organización del espacio no ha favorecido el buen desarrollo de la asignatura, sin embargo, se han sentido motivados ante el desarrollo de la dinámica $(59,1 \%)$ y les hubiese gustado conocer otras aplicaciones educativas $(81,8 \%)$.

\section{2.- Valoración del desarrollo del aprendizaje colaborativo}

\begin{tabular}{|l|c|c|c|c|}
\hline \multicolumn{1}{|c|}{ Ítems } & \multicolumn{2}{l|}{ Frecuencia } & \multicolumn{2}{l|}{$\begin{array}{c}\text { Porcentaje } \\
(100 \%)\end{array}$} \\
\cline { 2 - 5 } & $\mathrm{Si}$ & $\mathrm{No}$ & $\mathrm{Si}$ & No \\
\hline $\begin{array}{l}\text { ¿Opinas que el uso de las herramientas telemáticas } \\
\text { mejora o potencia el trabajo colaborativo? }\end{array}$ & 44 & 0 & 100 & 0 \\
\hline $\begin{array}{l}\text { ¿La interacción con el grupo de trabajo ha servido } \\
\text { para mejorar tu aprendizaje individualizado? }\end{array}$ & 44 & 0 & 100 & 0 \\
\hline
\end{tabular}

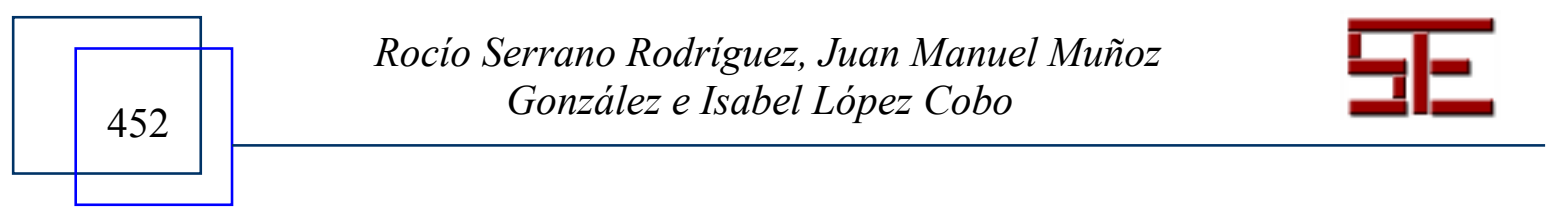




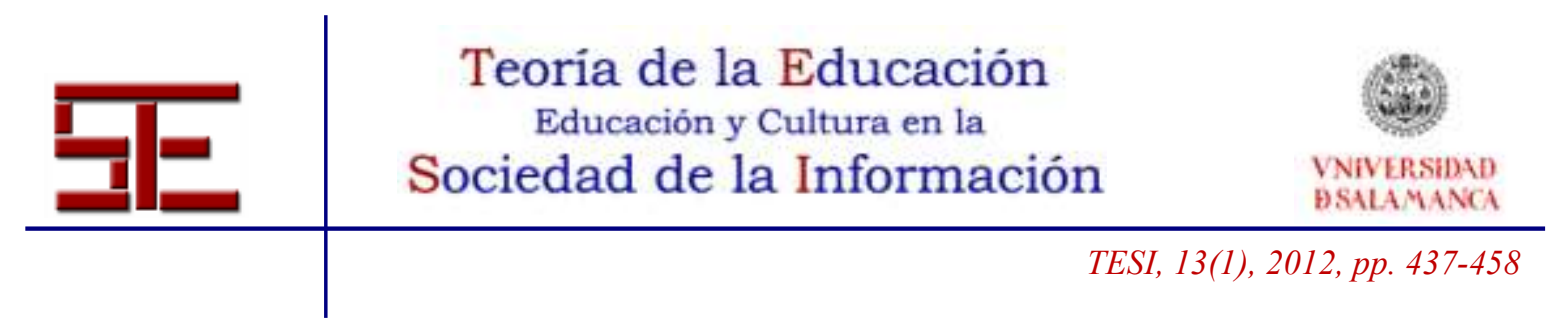

\begin{tabular}{|l|c|c|c|c|}
\hline $\begin{array}{l}\text { ¿Has adquirido nuevas habilidades y actitudes de } \\
\text { trabajo en equipo? }\end{array}$ & 44 & 0 & 100 & 0 \\
\hline $\begin{array}{l}\text { ¿Has intercambiado experiencias con otros } \\
\text { compañeros? }\end{array}$ & 44 & 0 & 100 & 0 \\
\hline $\begin{array}{l}\text { ¿El seguir una dinámica de aprendizaje colaborativo te } \\
\text { ha motivado? }\end{array}$ & 44 & 0 & 100 & 0 \\
\hline $\begin{array}{l}\text { ¿Ha incrementado tu capacidad de empatizar con los } \\
\text { compañeros/as? }\end{array}$ & 32 & 12 & 72,7 & 27,3 \\
\hline ¿Han mejorado las relaciones personales con el grupo? & 28 & 16 & 63,6 & 36,4 \\
\hline $\begin{array}{l}\text { ¿Con el trabajo cooperativo se desarrolla la seguridad } \\
\text { en uno mismo en el momento de compartir una idea? }\end{array}$ & 36 & 8 & 81,8 & 18,2 \\
\hline
\end{tabular}

Tabla 2: Frecuencias y porcentajes sobre la dinámica de aprendizaje colaborativo

En primer lugar apreciamos un conjunto de opiniones relacionadas con la influencia de la dinámica de aprendizaje cooperativo como una experiencia positiva en aspectos como: el uso de herramientas mejora o facilita el trabajo colaborativo (100\%), mejora el aprendizaje individualizado (100\%), se adquieren nuevas habilidades y actitudes de trabajo en equipo (100\%), mejoran las experiencias con los compañeros $(100 \%)$ y aumenta la motivación (100\%).

Por otro lado, los porcentajes menores se focalizan en aspectos personales como: las relaciones personales con el grupo $(63,3 \%)$, la capacidad de empatía $(72,7 \%)$ y la seguridad en uno mismo $(81,8 \%)$.

\section{6.- CONCLUSIONES}

El principal fin de la educación es desarrollar una enseñanza de calidad orientada a facilitar que nuestros alumnos aprendan bien, de modo que la calidad de la enseñanza debe ir ligada al desarrollo de estrategias educativas que ayuden a los alumnos a aprender significativamente, construir nuevos conocimientos y reflexionar sobre aquello que aprenden (Beltrán, 1993).

Tras la exposición de los resultados anteriores, deducimos que los alumnos valoran positivamente la innovación seguida en el desarrollo de la asignatura de "Diseño, desarrollo e innovación del currículum", así mismo, destacan muy positivo el uso de dinámicas de aprendizaje colaborativo. Aunque es razonable que consideren que el número de horas no es adecuado y como consecuencia no poder afianzar todos los

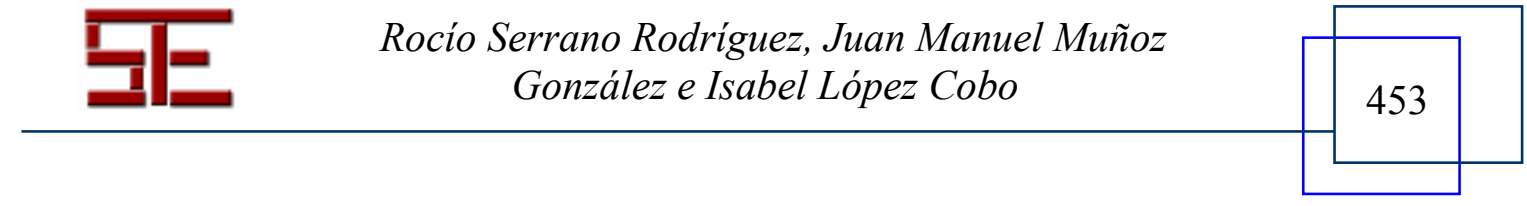




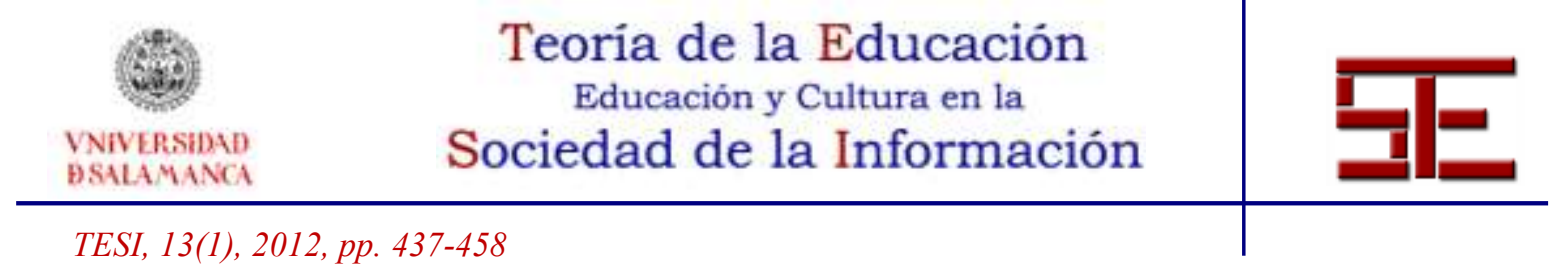

contenidos que esperaban. Por ello, en los resultados se manifiesta y se evidencia la necesidad de incorporar otras asignaturas donde se haga uso de estas herramientas y metodologías que mejorarían sus competencias como futuros docentes.

Somos conscientes de que para desarrollar óptimamente competencias TIC hace falta conjugar coherentemente la exposición cognitiva y la formación práctica. En cualquier caso, intentar incluir el desarrollo de tales competencias al terreno de la praxis nos obliga a revisar o replantear nuestra propia actividad como docentes, dado que "el alumno aprende sobre el profesor, además de aprender de él. Sea o no intencionado, el profesor se convierte en un modelo potencial de adulto o de profesor, y, si enseña a niños, es muy posible que estén tan interesados en él como en la materia" (Claxton, 1987).

Por otra parte, el aprendizaje colaborativo responde a una fuerte demanda social (Collazos, Mendoza, 2006) porque les facilita a los "aprendices" razonar acerca de la colaboración y crear un clima de trabajo adecuado fomentando la seguridad en uno mismo. Insistimos en que el trabajo grupal, además de permitir el desarrollo de actitudes positivas, habilidades y destrezas, contribuye a desarrollar y consolidar valores de solidaridad, compañerismo, cooperativismo y convivencia.

En este contexto, consideramos que la innovación metodológica que ha guiado el proceso de desarrollo de esta asignatura contribuye notablemente a mejorar el aprendizaje significativo de los contenidos educativos del proceso de formación y a desarrollar algunas de las competencias docentes que se han citado: familiarización con las TIC, trabajo en equipo y reflexión sobre los propios conocimientos del profesorado en formación (González, 2008).

Creemos, que es necesario utilizar tales recursos en educación tratando de aprovechar las grandes posibilidades que nos brindan actualmente estas herramientas para enriquecer los ambientes en los que se produce el aprendizaje (Piedrahita, 2006) y para favorecer el desarrollo de innovaciones docentes que aproximen el mundo de la investigación a la compleja realidad de las aulas (Trahtemberg, 2004). Desde esta perspectiva consideramos que la acción más urgente es fomentar el desarrollo de competencias docentes ligadas al uso práctico y motivador de tales recursos en la formación inicial del profesorado de todos los niveles, de modo que los futuros profesores se acostumbren a manejar tales instrumentos con familiaridad y los

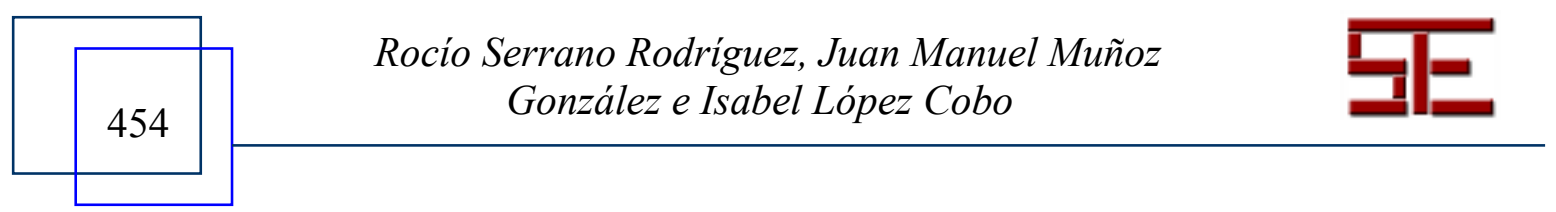




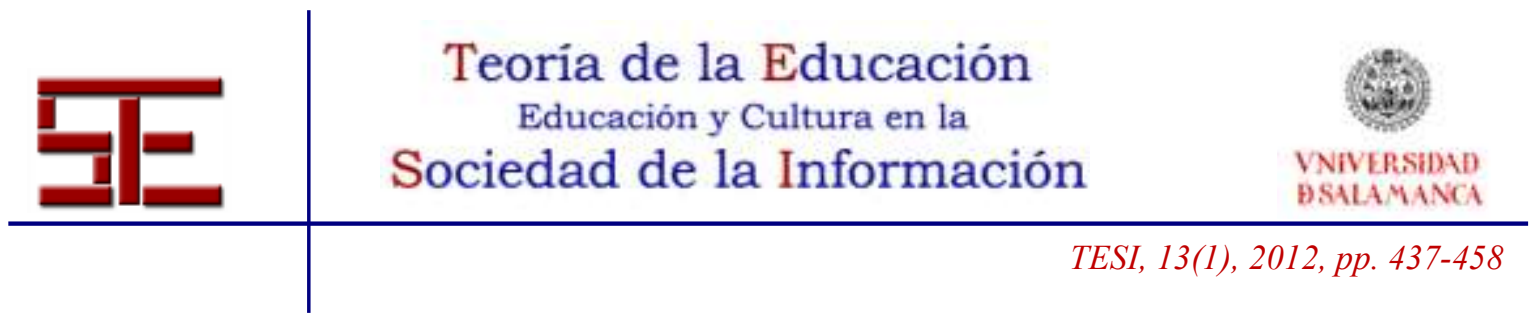

incorporen a su actividad educativa cuando accedan al ejercicio de la profesión docente (Pontes, Fuente y Pacheco, 2008).

También, pretendemos contribuir a mejorar la calidad de la educación y la investigación aportando datos relacionados con la autoevaluación del proceso de aprendizaje colaborativo, que los futuros profesores llevan a cabo al utilizar estas herramientas TIC en su formación.

Finalmente, somos conscientes de que el número de participantes en esta investigación no es suficiente como para considerar que los resultados obtenidos son generalizables, de modo que es necesario asumir con cautela los mismos y esperar al desarrollo de posteriores etapas del proyecto de trabajo que estamos llevando a cabo, en las que se recogerán datos de un número mayor de estudiantes.

\section{7.- BIBLIOGRAFÍA}

Area, M. (2005). Internet y la calidad de la educación superior en la perspectiva de la convergencia europea. Revista Española de Pedagogía, 63 (230), 85-100.

Beltrán, J. (1993). Procesos, estrategias y técnicas de aprendizaje. Madrid: Editorial Síntesis.

Cabero, J., Barroso, J. y Llorente., M.C. (2008). Plataforma de desarrollo de la tecnología educativa en Latinoamérica: un estudio a través de las aportaciones a sus congresos. Edutec: Revista de Tecnología Educativa, 25.

Cabero, J., Duarte, A. y Barroso, J. (1997). La piedra angular para la incorporación de los medios audiovisuales, informáticos y nuevas tecnologías en los contextos educativos: la formación y el perfeccionamiento del profesorado. Edutec: Revista de Tecnología Educativa, 8.

Cano, E. (2005). Cómo mejorar las competencias de los docentes. Barcelona: Graó.

Carrió, M. L. (2007). Ventajas del uso de la tecnología en el aprendizaje colaborativo. Revista Iberoamericana de Educación, 41 (3), 1-10.

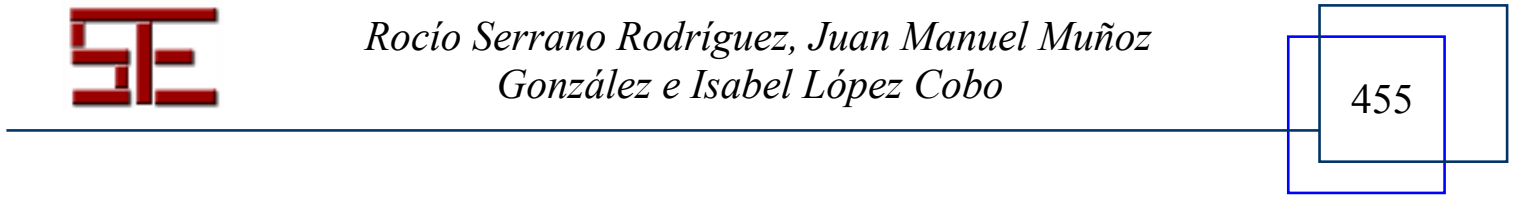




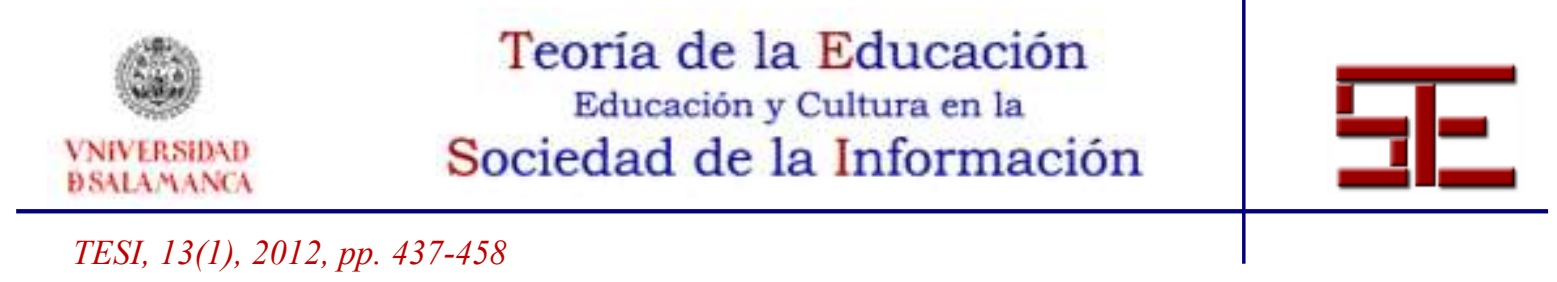

Cebrián, M. (1997). Nuevas competencias para la formación inicial y permanente del profesorado. Edutec: Revista de Tecnología Educativa, 6.

Claxton, G. (1987). Vivir y aprender. Madrid: Alianza.

Collazos, C.A. y Mendoza, J. (2006). Cómo aprovechar el aprendizaje colaborativo en el aula. Educación y Educadores, 9 (2), 61-76.

Da Pontes, J.P. (2004). Tecnologias de informação e comunicação na formação de professores: que desafios? Revista Iberoamericana de Educación, 24, 63-90.

Echazarreta, C., Prados, F., Poch, J. y Soler, J. (2009). La competencia "El trabajo colaborativo": una oportunidad para incorporar las TIC en la didáctica universitaria. Descripción de la experiencia con la plataforma ACME (UdG). UOC Papers: Revista sobre la Sociedad del Conocimiento, 8, 1-10.

Gisbert, M., Cela-Ranilla, J. e Isus, S. (2010). Las simulaciones en entornos TIC como herramienta para la formación en competencias transversales de los estudiantes universitarios. En De Pablos Pons, J. (Coord.). Buenas prácticas de enseñanza con TIC [monográfico en línea]. Revista Electrónica Teoría de la Educación: Educación y Cultura en la Sociedad de la Información, vol. 11, $\mathrm{n}^{\circ} 1$ (352-370). Universidad de Salamanca, Extraído el 27 de octubre, 2011, de http://revistatesi.usal.es/ revistas_trabajo/index.php/revistatesi/article/view/6309/ 6322.

González, F. (2008). El Mapa conceptual y el Diagrama V. Recursos para la Enseñanza Superior en el siglo XXI. Madrid: Narcea.

Johnson, D. (1998). Cooperation in the Classroom. Interaction Book company, 17.

León, O. y Montero, I. (1997). Diseño de Investigaciones. Introducción a la lógica de la investigación en Psicología y Educación. Madrid: McGraw-Hill.

Morales, E. M., Díaz, E. y García, F. J. (2011). Gestión de Objetos de Aprendizaje a través de la Red, basada en el desarrollo de competencias. En Hernández, M. J. y Fuentes, M. (Coords.). La red como recurso de información en educación. Revista Teoría de la Educación: Educación y Cultura en la Sociedad de la Información, 12, 1, 99-115. Extraído el 5 de octubre, 2011, de

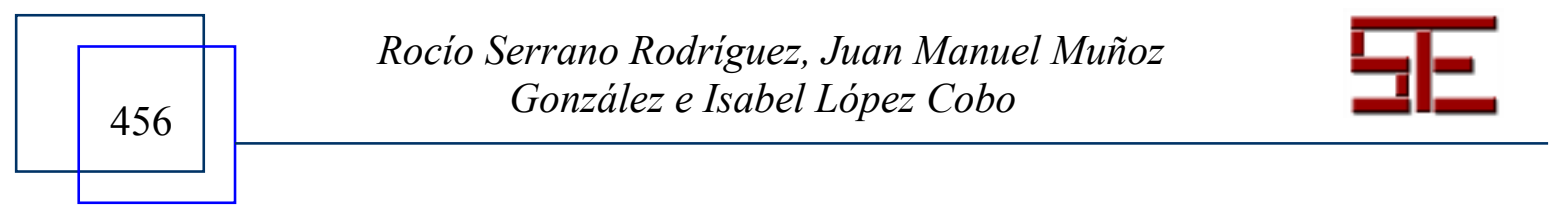




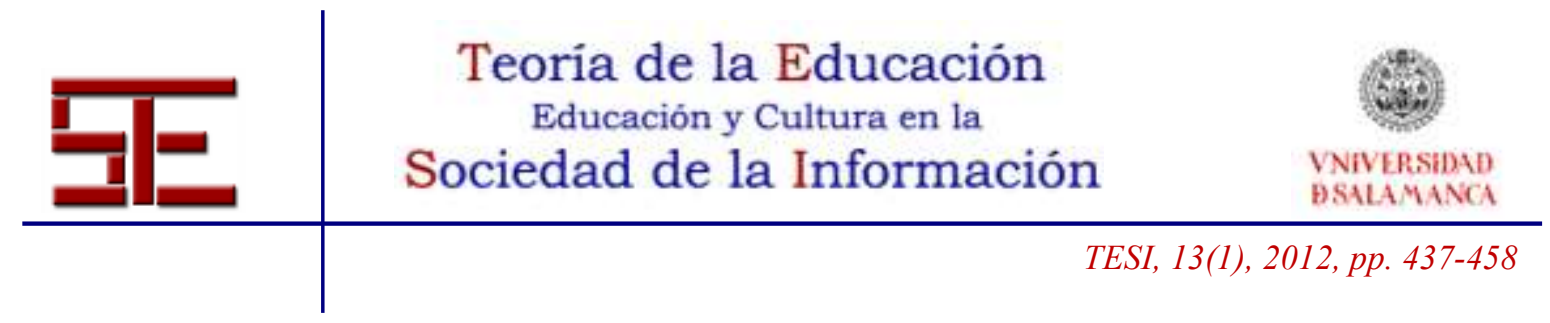

http://campus.usal.es/ revistas_trabajo/index.php/revistatesi/article/view/7825/78 52.

Ovejero, A. (1990). El aprendizaje cooperativo: Una alternativa a la enseñanza tradicional. Barcelona: P.P.U.

Peirano, C. y Domínguez, M.P. (2008). Competencia en TIC: el mayor desafío para la evaluación y el entrenamiento docente en Chile. Revista Iberoamericana de Evaluación Educativa, 1 (2), 106-124.

Piedrahita, F. (2006). Un modelo para integrar las TICs en el currículo. Extraído el 5 de octubre de 2011, de http://www.eduteka.org/ EdutekaRibie.php.

Pontes, A. (2005). Aplicaciones de las nuevas tecnologías de la información en la educación científica. $1^{\mathrm{a}}$ Parte: Funciones y recursos. Revista Eureka sobre Enseñanza y Divulgación de las Ciencias, 2 (1), 2-18. Extraído el 25 de Septiembre, 2011 de http://www.apac-eureka.org/revista/.

Pontes, A., Fuente, M. y Pacheco, M. (2008). Tecnologías de la información y la comunicación en la enseñanza secundaria. En A. Pontes [Coord.], Aspectos generales de la formación psicopedagógica del profesorado de educación secundaria (pp. 268-308).

Rosario, H. (2008). La Web. Herramienta de trabajo Colaborativo: Experiencia en la Universidad de Carabobo. Pixel-Bit: Revista de Medios y Educación, 31, 131139.

Sánchez, L., Lombardo, J. M., Riesco, M. y Joyanes, L. (2004). Las TICs y la formación del profesorado en la Enseñanza Secundaria. Revista: Educación y Futuro Digital. Extraído el 4 de abril, 2004 de http://www.cesdonbosco.com/ revista/ artículos2004/.

Stahl, G., Koschmann, T. \& Suthers, D. (2006). Computer-supported collaborative learning. In R. K. Sawyer (Ed.), Cambridge handbook of the learning sciences. Cambridge, UK: Cambridge University Press. Extraído el 25 de enero, 2008 de http://www.ischool.drexel.edu/faculty/gerry/cscl/csclspanish.htm.

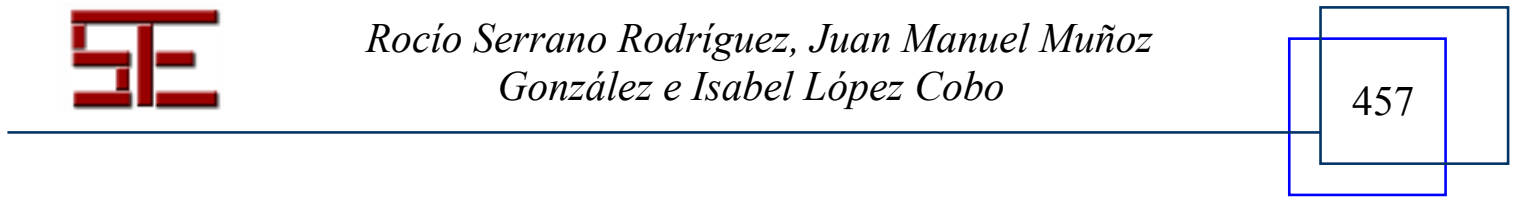




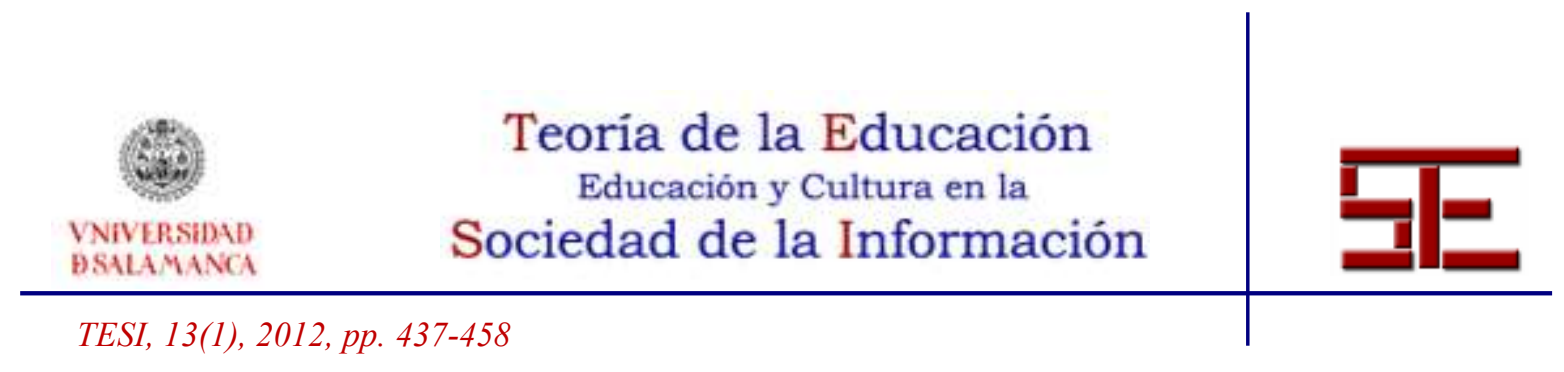

Trahtemberg, L. (2004). El impacto previsible de las nuevas tecnologías en la enseñanza y la organización escolar. Revista Iberoamericana de Educación, 24, 37-62.

Villalustre, L. y Del Moral, M. E. (2011). Webquest y wikis: búsqueda de información en red y desarrollo de competencias en colaboración. En Hernández Serrano M.J. y Fuentes Agustí (Coords.). La red como recurso de información en educación. Revista Teoría de la Educación: Educación y Cultura en la Sociedad de la Información, vol. 12, n ${ }^{\circ}$ 1. Universidad de Salamanca, 190-208. Extraído el 10 de septiembre, 2011, de http://campus.usal.es/ revistas_trabajo/index.php/ revistatesi/article/view/7829/7856.

Para citar el presente artículo puede utilizar la siguiente referencia:

Serrano Rodríguez, R., Muñoz González, J. M. y López Cobo, I. (2012). Valoración por los estudiantes de la licenciatura en psicopedagogía, de una experiencia innovadora basada en estrategias de aprendizaje colaborativo y el uso del courselab. Revista Teoría de la Educación: Educación y Cultura en la Sociedad de la Información. 13(1), 437458 [Fecha de consulta: $\mathrm{dd} / \mathrm{mm} / \mathrm{aaaa}]$.

http://campus.usal.es/ revistas_trabajo/index.php/revistatesi/article/view/8820/9024

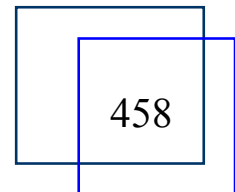

Rocío Serrano Rodríguez, Juan Manuel Muñoz González e Isabel López Cobo 